\title{
Three Dimensional Analyses of Degradation in PEMFCs
}

\author{
Takeshi Daio $^{1}$, Katsuaki Suganuma ${ }^{1}$ and Ichihito Narita ${ }^{2}$ \\ 1. The Institute of Scientific and Industrial Research, Osaka University, Ibaraki, Osaka, Japan \\ 2. Department of Technology Education, Osaka Kyoiku University, Kashiwara, Osaka, Japan
}

Proton exchange membrane fuel cell (PEMFC) is one of the candidate materials which can utilize the hydrogen without emitting greenhouse effect gas. However, the lifetime of PEMFCs is still short due to the degradation phenomena during operation. Previous studies suggest that applied temperature leads aggregation of supported particles and changes the distribution of metal nanoparticles and ionomers on carbon blacks. Recently, nanoscale distribution of these nanoparticles and ionomers attracts much attention to clarify these degradation mechanisms of PEMFCs and to improve the performance. Recent studies of distribution of the nanoparticles and ionomers of PEMFCs were investigated by tomography [1] and in-situ observation [2].

To date, tomography and in-situ observation have been individually conducted to observe the dynamic change of morphology. In particularly, on the PEMFCs, tomography (which was taken without heating) archived to distinguish between pure noble metal nanoparticles, ionomers and carbon blacks in an image by using the Z-contrast. And the catalytic behaviours were visualised with in-situ to simulate degradation. However, tomography with in-situ observation of PEMFCs has not been achieved because of the size limitation of the sample heating holder and the chamber. In fact, several heating holders are proposed, but these are difficult to apply for electron tomography because of tiny spaces around the objective pole piece and thickness of the holder which results in small tilt angle e.g. 25 degree. Also, heater of the holder makes missing angle. In addition, in-situ observation of PEMFCs with heating is difficult since the supported particles change the size and distribution due to reaction between nanoparticles, ionomers and carbon blacks. Therefore, thinner heating unit and holder tip is needed. Also, conventional heating holder has a heating unit made by wires. Therefore, it is important to quantitatively identify the temperature as much as possible to avoid over shoot of temperature.

In this study, MEMS holder (Nano Ex/iv, FEI) was employed to perform stable analysis while keeping temperature within 1 degree to clarify the difference of each state of PEMFCs quantitatively. Also, quadrant detector combined with low profile holder enabled the time series of in situ EDX electron tomography. PEMFCs were prepared using Pt/Co catalysis nanoparticle and Ionomer (Nafion) was dispersed on the carbon black. At $393 \mathrm{~K}$, the specimen was kept for 60 minutes to observe the time dependency. Figure 1 shows the STEM-HAADF images of the PEMFCs at $353 \mathrm{~K}$ and $393 \mathrm{~K}$. After the heating, morphological change of catalytic nanoparticles was confirmed. This is consistent with previous studies. However, some nanoparticles which are embedded in the Nafion seem to keep the shape. Similar behavior has been reported in different materials [3]. The Nafion distribution is also confirmed in STEM-HAADF images (white arrows). The result shows that Nafion distribution changes as increasing temperature. This shows change of three phase boundaries which is important to reveal the degradation mechanism of PEMFCs [4]. 
References:

[1] Matsumoto, H. et al, Microscopy and Analysis (2013), p. 13.

[2] Lopez-Haro, M. et al, Nature communications 5 (2014)

[3] Khalavka, Y. et al, The Journal of Physical Chemistry C 111(2007), p. 12886.

[4] The authors acknowledge funding from the MEXT/JSPS KAKENHI Grant Number Young Scientists (B) (No. 15K17443)

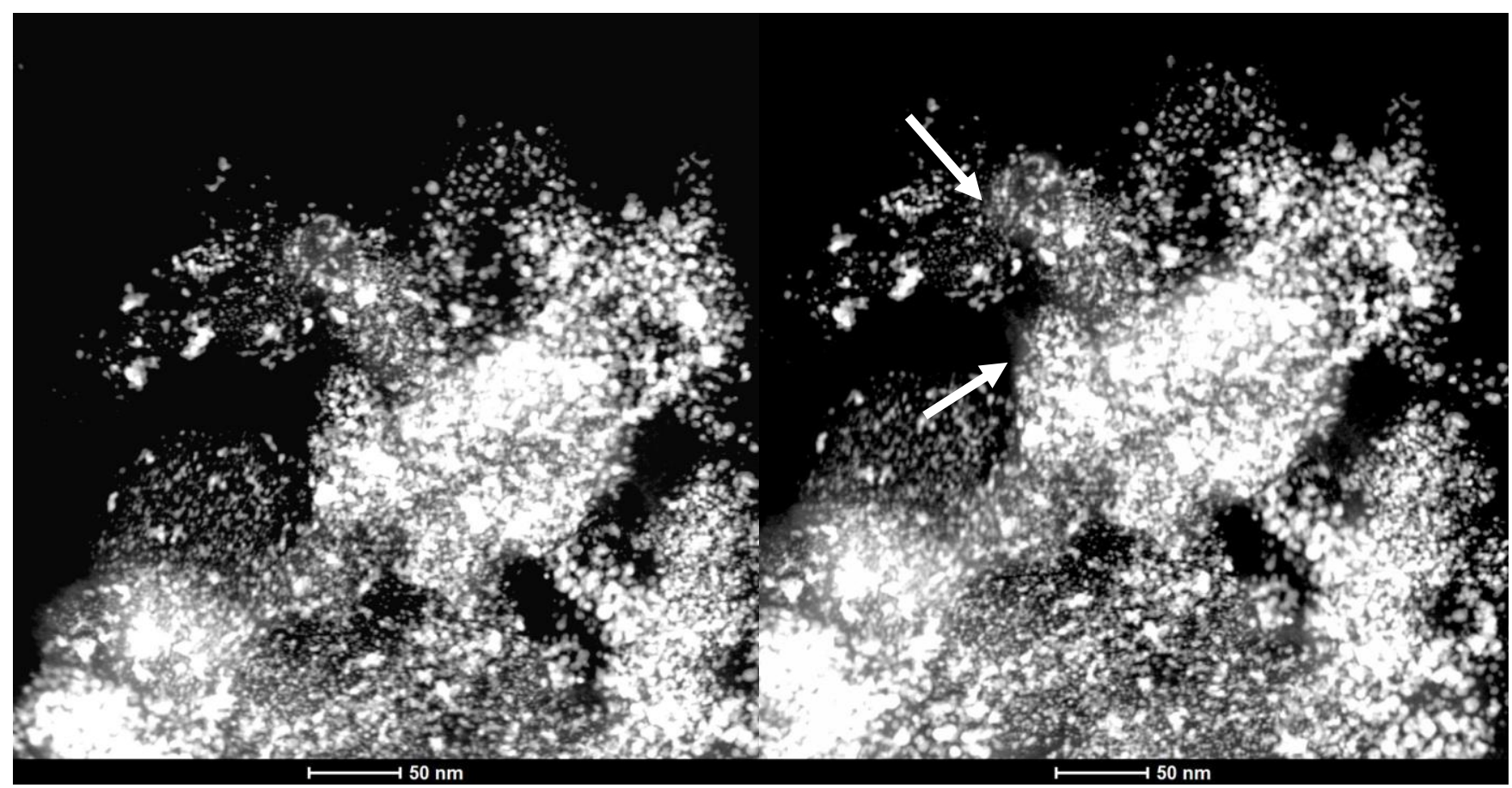

Figure 1. STEM-HAADF images of (a) PEMFCs at $353 \mathrm{~K}$ and (b) $393 \mathrm{~K}$, respectively. 